\title{
Chickenpox and asymptomatic COVID-19 after first cycle of alemtuzumab for multiple sclerosis
}

\author{
Mario Habek ${ }^{1,2}$ iD
}

Received: 27 April 2021 / Accepted: 12 July 2021 / Published online: 31 July 2021

(c) Fondazione Società Italiana di Neurologia 2021

A 28-year-old woman, kindergarten teacher, developed double vision on left gaze in May 2018. Neurological examination revealed right internuclear ophthalmoplegia. Brain and spinal cord MRI showed 15 T2 and FLAIR hyperintense periventricular, juxtacortical, and infratentorial lesions and 2 lesions in the cervical spinal cord; 2 brainstem lesions showed Gadolinium enhancement. Cerebrospinal fluid was positive for oligoclonal IgG bands, and she was diagnosed with relapsing remitting multiple sclerosis (RRMS). The patient fully recovered after pulse methylprednisolone treatment. In July 2018, she developed incomplete transverse myelitis with Th12 sensory level when she again received pulse methylprednisolone treatment. MRI performed in February 2019 showed 5 new supratentorial lesions. Because of the highly active RRMS and pregnancy planning, it was decided to start treatment with alemtuzumab. During the work-up before starting alemtuzumab, varicella zoster virus (VZV) IgG came back negative, and vaccination against VZV was recommended before starting treatment. However, it took three doses of live attenuated varicella virus (OKA strain) vaccine (Varilrix ${ }^{\circledR}$ ) until a titer of VZV IgG was satisfactory for treatment start $(0.87)(<0.60$ negative; $0.60-<0.90$ intermediate; $\geq 0.90$ positive; enzyme linked fluorescent assay). From March 9 to 10, 2020, the patient received the first course of alemtuzumab. She developed a rash from day 2 through 5 , which was symptomatically treated. The patient did not take any other concomitant treatments. Lymphocytes ranged from 0.32 in May 2020 to $0.88 \times 10^{9}$ in October 2020. After an outbreak of chickenpox in the kindergarten, the patient developed small, itchy

Mario Habek

mhabek@mef.hr

1 Department of Neurology, Referral Center for Autonomic Nervous System Disorders, University Hospital Center Zagreb, Kišpatićeva 12, 10000 Zagreb, Croatia

2 School of Medicine, University of Zagreb, Zagreb, Croatia blisters on the arms and back on November 20, 2020 (Fig. 1). Therapy with acyclovir, $400 \mathrm{mg} 5$ times a day through 7 days, was initiated, and she completely recovered. At the same time, her husband was diagnosed with COVID-19, and she spent 14 more days in self isolation. She never developed any symptoms characteristic for COVID-19, and she returned to work. Oropharyngeal swab during her husband's quarantine was not performed. In December 2020, serology for SARS-CoV-2 revealed the titer of antibody to spike protein titer of $102 \mathrm{U} / \mathrm{ml}$ (positive value $\geq 0.8 \mathrm{U} / \mathrm{ml}$, Elecsys ${ }^{\circledR}$ anti-SARSCoV-2 S assay, Roche Diagnostics Int., Rotkreuz, Switzerland).In March 2021, VZV IgG titer was 3.89, and the patient received second cycle of alemtuzumab.

Alemtuzumab is an anti-CD52 humanized monoclonal antibody which, by depletion and consecutive repopulation of $\mathrm{T}$ and $\mathrm{B}$ cells, in many persons with RRMS leads to a long-lasting remission [1]. One of the possible complications associated with alemtuzumab is increased risk of infections, the most severe one usually happening in close temporal relation to alemtuzumab infusions. The majority of the infections reported were caused by herpesviruses; however, with more patients being exposed, reports of listeriosis and other rare bacterial, viral, or fungal infections after an alemtuzumab treatment cycle have emerged [2]. Based on this increased risk of infections, many neurological societies recommended against starting or continuing alemtuzumab during the COVID-19 pandemic [3]. However, initial reports indicated mild COVID-19 infection in persons with RRMS receiving alemtuzumab [4]. Another problem of high efficacy RRMS therapy is possibility of immune system to mount a response to an infectious agent or vaccination. Only limited evidence regarding this question exists on alemtuzumab. In a small study, serum antibodies against common viruses remained detectable after treatment, and vaccine responses were normal to $\mathrm{T}$ cell-dependent recall antigens (tetanus, diphtheria, and polio), a $\mathrm{T}$ cell-dependent novel antigen (meningococcus $\mathrm{C}$ ), and $\mathrm{T}$ 
Fig. 1 Small, itchy blisters on the arms and back typical for chickenpox

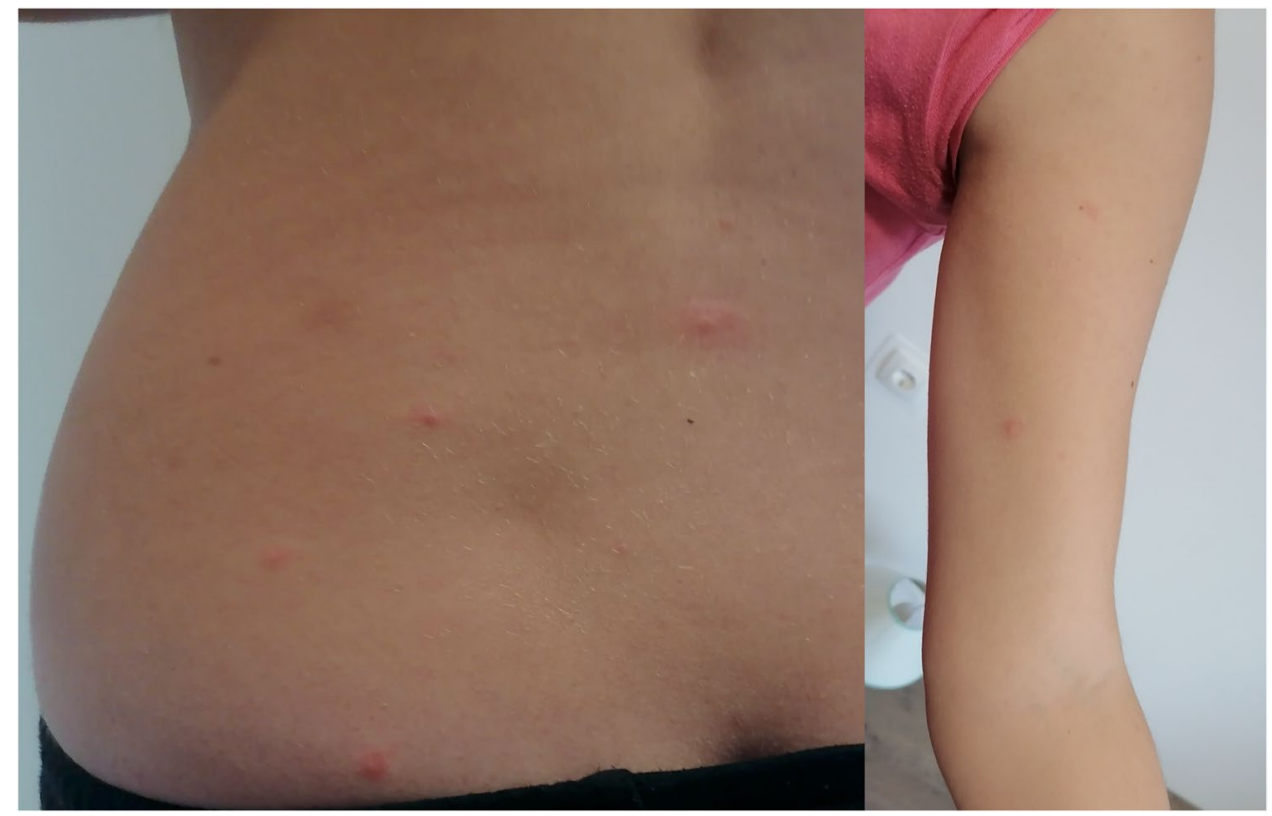

cell-independent antigens (pneumococcal) [5]. Furthermore, there was no evidence for a diminished response to vaccinations in 5 patients studied within 6 months of alemtuzumab treatment [5]. So far, three cases of COVID-19 occurring in persons with RRMS from 1 week to 11 months after the last alemtuzumab cycle with positive SARS-CoV-2 IgG antibody were reported [6, 7].

The presented case had coexistent asymptomatic COVID-19 infection and very mild chickenpox 7 months after receiving the first cycle of alemtuzumab. The VZV vaccine effectiveness in a meta-analysis was $92 \%(95 \%$ CI: 88-95\%), explaining the very mild clinical manifestation of chickenpox in the presented patient [8]. Furthermore, recently, a series on varicella-like exanthema as a specific COVID-19-associated cutaneous clinical picture has been described [9]. While the possibility that cutaneous manifestations in the presented case could be a consequence of COVID-19 cannot be excluded, the fourfold increase in the VZV IgG titer strongly argues for VZV infection. Finally, the main limitation of this case is that one cannot be certain that the patient developed the SARS CoV2 antibodies exactly during her chickenpox infection and her husband SARS CoV2 infection, although this is highly likely.

This case, together with previously published ones, suggests that appropriately selected persons with highly active RRMS benefit from treatment with alemtuzumab outweighs the risk, even in the time of COVID-19 pandemic.

Author contribution Study concept and design; acquisition of data; analysis and interpretation of data; drafting of the manuscript; critical revision of the manuscript for important intellectual content; administrative, technical, and material support: Habek.

\section{Declarations}

Ethical approval Not applicable.

Informed consent Not applicable.

Competing interests $\mathrm{MH}$ : Participated as a clinical investigator and/or received consultation and/or speaker fees from the following: Biogen, Sanofi Genzyme, Merck, Bayer, Novartis, Pliva/Teva, Roche, Alvogen, Actelion, Alexion Pharmaceuticals, and TG Pharmaceuticals.

\section{References}

1. Willis MD, Harding KE, Pickersgill TP, Wardle M, Pearson OR, Scolding NJ, Smee J, Robertson NP (2016) Alemtuzumab for multiple sclerosis: long term follow-up in a multi-centre cohort. Mult Scler 22:1215-1223

2. Winkelmann A, Loebermann M, Reisinger EC, Hartung HP, Zettl UK (2016) Disease-modifying therapies and infectious risks in multiple sclerosis. Nat Rev Neurol 12:217-233

3. Giovannoni G, Hawkes C, Lechner-Scott J, Levy M, Waubant E, Gold J (2020) The COVID-19 pandemic and the use of MS disease-modifying therapies. Mult Scler Relat Disord 39:102073

4. Carandini T, Pietroboni AM, Sacchi L, De Riz MA, Pozzato M, Arighi A, Fumagalli GG, Martinelli Boneschi F, Galimberti D, Scarpini E (2020) Alemtuzumab in multiple sclerosis during the COVID-19 pandemic: a mild uncomplicated infection despite intense immunosuppression. Mult Scler 26:1268-1269

5. McCarthy CL, Tuohy O, Compston DA, Kumararatne DS, Coles AJ, Jones JL (2013) Immune competence after alemtuzumab treatment of multiple sclerosis. Neurology 81:872-876 
6. Fernández-Díaz E, Gracia-Gil J, García-García JG, Palao M, Romero-Sánchez CM, Segura T (2020) COVID-19 and multiple sclerosis: a description of two cases on alemtuzumab. Mult Scler Relat Disord 45:102402

7. Fiorella C, Lorna G (2020) COVID-19 in a multiple sclerosis (MS) patient treated with alemtuzumab: insight to the immune response after COVID. Mult Scler Relat Disord 46:102447

8. Marin M, Marti M, Kambhampati A, Jeram SM, Seward JF (2016) Global varicella vaccine effectiveness: a meta-analysis. Pediatrics 137:e20153741
9. Marzano AV, Genovese G, Fabbrocini G, Pigatto P, Monfrecola G, Piraccini BM, Veraldi S, Rubegni P, Cusini M, Caputo V, Rongioletti F, Berti E, Calzavara-Pinton P (2020) Varicella-like exanthem as a specific COVID-19-associated skin manifestation: multicenter case series of 22 patients. J Am Acad Dermatol $83: 280-285$

Publisher's Note Springer Nature remains neutral with regard to jurisdictional claims in published maps and institutional affiliations. 